\title{
A Novel Service Composition Approach for Application Migration to Cloud
}

\author{
Xianzhi Wang ${ }^{1,2}$, Xuejun Zhuo ${ }^{1}$, Bo Yang ${ }^{1}$, Fan Jing Meng ${ }^{1}, \mathrm{Pu} \mathrm{Jin}^{1}$, Woody Huang ${ }^{3}$, \\ Christopher C. Young ${ }^{3}$, Catherine Zhang ${ }^{3}$, Jing Min Xu ${ }^{1}$, and Michael Montinarelli ${ }^{4}$ \\ ${ }^{1}$ IBM Research - China, Beijing, P.R. China \\ $\{$ wxianzhi, zhuoxuej, yangbbo, mengfj, jinpubj, xujingm\}@cn.ibm.com \\ ${ }^{2}$ Harbin Institute of Technology, Harbin, Heilongjiang, P.R. China \\ xianzhi.wang@hit.edu.cn \\ ${ }^{3}$ IBM Research - Watson, Yorktown, NY, US \\ \{ywh, ccyoung, cxzhang\}@us.ibm. com \\ ${ }^{4}$ IBM Global Technology Services, Rochester, NY, US \\ mmonti@us.ibm.com
}

\begin{abstract}
Migrating business applications to cloud can be costly, laborintensive, and error-prone due to the complexity of business applications, the constraints of the clouds, and the limitations of existing migration techniques provided by migration service vendors. However, the emerging software-as-aservice offering model of migration services makes it possible to combine multiple migration services for a single migration task. In this paper, we propose a novel migration service composition approach to achieve a costeffective migration solution. In particular, we first formalize the migration service composition problem into an optimization model. Then, we present an algorithm to determine the optimal composition solution for a given migration task. Finally, using synthetic trace driven simulations, we validate the effectiveness and efficiency of the proposed optimization model and algorithm.
\end{abstract}

Keywords: Cloud computing, modeling, migration, service composition.

\section{Introduction}

The increasingly wide adoption of cloud computing has witnessed huge demands for migrating business applications to cloud. In 2012, IDC reported that more than $50 \%$ of European larger companies stated that they have a strategy for IT infrastructure refactoring to benefit from cloud economics, of which the cloud migration investment is forecast to grow at $30.6 \%$ Compound Annual Growth Rate in the next 5 years [1].

In response to the huge migration demands, many migration service vendors have emerged to offer diverse migration services. They can be categorized into three major types: image-based migration, application-centric migration, and migration to virtualized containers. The image-based migration technique, such as Racemi® [2] and CohesiveFT [3], converts source serves into virtual images and imports them into target cloud after necessary adjustments. Application-centric migration, such as 
AppZero [4] and CliQr ${ }^{\mathrm{TM}}$ [5], provisions a new application deployment environment on the target by extracting and migrating the application artifacts, configurations, and resources from the source. The migration to virtualized containers method, such as Ravello [6] and CohesiveFT Software Defined Networking [3], migrates the source VM without any modification to run in a virtualized container inside the target cloud.

However, migrating business applications to cloud is considered costly, laborintensive, and error-prone due to the complexity of the applications, the constraints of the clouds, and the limitations of existing migration techniques provided by migration service vendors[7]. It is extremely difficult or even impossible for single vendor to migrate a complex application entirely on its own. Fortunately, the emerging software-as-a-service model makes it possible to combine multiple migration services to accomplish a single migration task. By consolidating the diverse migration capabilities provided by multiple vendors, a more comprehensive and flexible migration service can be provided. For example, Racemi ${ }^{\circledR}$ can provide server migration services to the IBM® SmartCloud ${ }^{\circledR}$ Enterprise (SCE)[8] but cannot remediate middleware and application configurations (e.g. hostname, IP address) after migration; whereas the CohesiveFT can close the gap with the network virtualization service. As can be seen, the application can be successfully migrated to IBM® SCE by composing the services provided by the two vendors.

In this paper, we propose a novel service composition approach to provide an application migration solution with a wide-spectrum of capabilities, by consolidating diverse migration techniques offered by different vendors. The main contributions of the paper are two-fold: 1) We formalize the migration service composition problem into an optimization model; 2) We provide an algorithm to solve the optimization problem and validate it using simulations.

The remainder of this paper is organized as follows. Section 2 introduces the modeling of the basic concepts and the problem. The matching and optimization algorithm is presents in Section 3 and evaluated in Section 4. Section 5 investigates related work. Section 6 concludes the paper and discusses the future works.

\section{Problem Definition and Modeling}

In migration context, a business application can be modeled as state with a hierarchy of components with valued configuration attributes and interdependencies. The problem of determining an optimal cost-effective migration solution can be considered as a state transition problem, aiming to find an optimal subset of vendor services and their operation sequence so that they can collaboratively transform the application from its initial state to a cloud-acceptable state.

\subsection{Problem Definition}

In this section, we consider scenarios where applications are migrated into a single given cloud and formalize the basic concepts as follows. 
$c\left(a_{1}, a_{2}, \ldots, a_{k}\right):$ denotes an application component which can be migrated separately as an individual unit, where $\forall a_{i}, i \in\{1, \ldots, k\}$ are the detailed configuration attributes attached to the component.

$\tilde{c}\left(a_{1}=x_{1}, a_{2}=x_{2}, \ldots, a_{k}=x_{k}\right):$ denotes an instance of component $c$ in which $\forall a_{i}, i \in\{1, \ldots, k\}$ have been set with specific values.

$C=\left\{c_{1}, c_{2}, \ldots, c_{l}\right\}:$ denotes all the components to be migrated in the application.

$S\left(C^{*}\right)=\left(\tilde{C}^{*}, D\right)$ : denotes a state of a set of application components $C^{*} \subseteq C$. $\tilde{C}^{*}=\left\{\tilde{c}_{1}^{*}, \tilde{c}_{2}^{*}, \ldots, \tilde{c}_{g}^{*}\right\}$, which represents an instance of this set of components where each component is attached with valued configuration attributes. $D=\left\{\left(\right.\right.$ type $\left.\left., c_{\text {from }} \rightarrow c_{\text {to }},\left\{c_{\text {from }} . a_{x} \rightarrow c_{t o} . a_{y}\right\}\right)\right\}$, which denotes the dependency relations between components. The first parameter indicates the dependency type, e.g., runsOn, connectsTo, includes, etc. The second parameter indicates the two components related to this dependency. The last parameter indicates the detailed mapping relations between the attributes of the corresponding components, if any.

Based on above definition, the problem-specific concepts are formalized as below: $S_{i n i}(C)=\left(\tilde{C}_{i n i}, D_{i n i}\right):$ denotes the initial state of the application to be migrated.

$\mathbf{S}_{t g t}(C)=\left\{S_{t g t}^{1}(C), S_{t g t}^{2}(C), \ldots, S_{t g t}^{k}(C)\right\}$ : denotes a collection of all possible acceptable target states of the application in the cloud, where $S_{t g t}^{i}(C)=\left(C_{t g t}^{i}, D_{t g t}^{i}\right)$ is an individual state in it.

$V=\left[v_{1}, v_{2}, \ldots, v_{n}\right]:$ denotes a collection of migration services provides by vendors.

$\mathbf{M}=\left[M_{1}, M_{2}, \ldots, M_{n}\right]$ : denotes vendors' migration capability. More specifically, the capability of each vendor $v_{j}(\in V)$ is represented by its enabled state transition $M_{j}=\left(S_{j}^{\text {cond }}\left(C_{j}^{\text {cond }}\right), S_{j}^{\text {in }}\left(C_{j}^{\text {mig }}\right), S_{j}^{\text {out }}\left(C_{j}^{\text {mig }}\right)\right)$, where $C_{j}^{\text {mig }}$ is the component to be migrated by $v_{j}, S_{j}^{\text {in }}\left(C_{j}^{\text {mig }}\right)$ and $S_{j}^{\text {out }}\left(C_{j}^{\text {mig }}\right)$ are the input and output states of $C_{j}{ }^{\text {mig }}$, respectively, while $S_{j}^{\text {cond }}\left(C_{j}^{\text {cond }}\right)$ is the requirements imposed by $v_{j}$ as prerequisites on components other than $C_{j}{ }^{\text {mig }}\left(\neq C_{j}{ }^{\text {cond }}\right)$. Note that a vendor can be matched to multiple components within single migration task. For example, a vendor which performs server-based migration may possibly be mapped to two separate servers (regarded as different components), respectively.

$\operatorname{Cost}=\left[\operatorname{Cost}\left(v_{1}\right), \operatorname{Cost}\left(v_{2}\right), \ldots, \operatorname{Cost}\left(v_{n}\right)\right]:$ denotes vendor-specific functions for calculating migration cost, which are specific to the number of migrated components plus operational cost.

$\mathbf{H}=\left[H\left(v_{1}\right), H\left(v_{2}\right), \ldots, H\left(v_{n}\right)\right]$ : denotes the quantified measurement of human efforts required by vendor services per usage based on historical experiences.

$\mathbf{T}=\left[T\left(v_{1}\right), T\left(v_{2}\right), \ldots, T\left(v_{n}\right)\right]:$ denotes the predicted time consumed by each vendor to perform migration per usage based on benchmark.

$H_{\max }$ : denotes the maximal human effort acceptable for application migration

$T_{\max }$ : denotes the maximal migration time acceptable for application migration 
$\mathbf{G}=(\mathbf{N}, \mathbf{E})$ : is a directed acyclic graph which denotes a migration solution. $\mathbf{N}=\left[N_{1} v_{1}, N_{2} v_{2}, \ldots, N_{n} v_{n}\right]$ is a set of nodes, i.e., a repetitive set of vendors which are selected to collaboratively accomplish the migration task, $N_{i} \in\{0,1, \ldots, n\}$. $\mathbf{E}=\left\{\left(v_{x}, v_{y}, P_{x, y}\right)\right\}$ is a set of edges, i.e., directed links (e.g., from $v_{x}$ to $v_{y}$ ) representing their precedent order in migration, and context information $P_{x, y}$.

\subsection{Problem Modeling}

The objective of the migration problem is to determine an optimal migration solution denoted as a graph $\mathbf{G}$ which minimizes the overall migration and time-framed operational cost under user specified constrains on time and human efforts. The optimization problem is formally formulized as follows:

$$
\begin{array}{cc}
\arg \min _{\mathbf{G}} \sum_{i=1}^{n} N_{i} \operatorname{Cost}\left(v_{i}\right) \\
\text { s.t. } & f\left(S_{i n i}(C), \mathbf{S}_{t g t}(C), \mathbf{M}, \mathbf{G}\right)=1 \\
& \sum_{i=1}^{n} N_{i} H\left(v_{i}\right) \leq H_{\max } \\
& t_{\max }(\mathbf{G}, \mathbf{T}) \leq T_{\max }
\end{array}
$$

In Eq (2), $f=1$ if and only if $\mathbf{G}$ is a feasible migration solution, i.e., it is able to transform application state from $S_{i n i}$ to an acceptable target state in $\mathbf{S}_{t g t}$, by collaboratively utilizing vendors' migration capability represented by $\mathbf{M}$. In Eq (4), $t_{\max }(\mathbf{G}, \mathbf{T})$ is the maximum migration time for solution $\mathbf{G}$ estimated based on $\mathbf{T}$.

\section{The Algorithm}

The migration problem defined in the last section can be reduced to the Subset Sum problem with exponential set size which is proved to be NP-Hard. In this paper, we propose an algorithm based on A-star algorithm with effective pruning strategies to significantly reduce the solution space.

Firstly, we will introduce how to calculate Lower-Bound of Potential Cost (LBPC) for each intermediate state. In particular, given any intermediate state $S$ and a target state $S_{t g t}^{i}$, the corresponding LBPC is calculated as the maximum LBPC estimated for each attribute of each component of the given application. Suppose $A$ is a set of all attributes of the application, $x(a)$ and $x_{t g t}(a)$ are the current and target values of $a(\in A)$ specified in $S(C)$ and $S_{t g t}^{i}(C)$, respectively. We define:

$$
\operatorname{Cost}^{*}\left(S(C), S_{t g t}^{i}(C)\right)=\max _{a \in A} \operatorname{Cost}^{*}\left(a: x \rightarrow x_{t g t}\right)
$$

where $\operatorname{Cost}^{*}\left(x(a) \rightarrow x_{t g t}(a)\right)$ is the estimated LBPC for transforming the value of a single attribute $a$ into a given target value, which can be further calculated as the 
minimum value among the minimal cost of value-change achieved directly by a single vendor, and minimal cost achieved by multiple vendors.

$$
\operatorname{Cost}^{*}\left(a: x \rightarrow x_{t g t}\right)=\min \left(\operatorname{Cost}_{i d c}\left(a: x \rightarrow x_{t g t}\right), \operatorname{Cost}_{d c}\left(a: x \rightarrow x_{t g t}\right)\right)
$$

Cost $_{i d c}$ contains at least two parts, the minimal cost for any vendor that accepts the given attribute value, and the minimal cost for any vendor that can transform the attribute into target value.

$$
\operatorname{Cost}_{i d c}(a)=\text { Cost }_{\text {fromX }}+\text { Cost }_{\text {toTgt }}
$$

For the value-change performed by single vendor, the LBPC is the minimal cost of all vendors that enables the value-change, i.e.,

$$
\operatorname{Cost}_{d c}(a)=\min \left\{\operatorname{Cost}(v) \mid v \text { enables } a: x \rightarrow x_{t g t}\right\}
$$

Also $\operatorname{Cost} *\left(S, S_{t g t}^{i}\right)$ should be set $+\infty$ when any target value is unreachable.

The input are the initial and target states: $S_{i n i}$ and $\mathbf{S}_{t g t}$, registered vendors $V$ represented with $(\mathbf{M}, \mathbf{C o s t}, \mathbf{H}, \mathbf{T})$, and user specified constraints: $H_{\max }$ and $T_{\max }$. The output is the $\mathbf{G}=(\mathbf{N}, \mathbf{E})$ as defined above. The detail procedure is as follows.

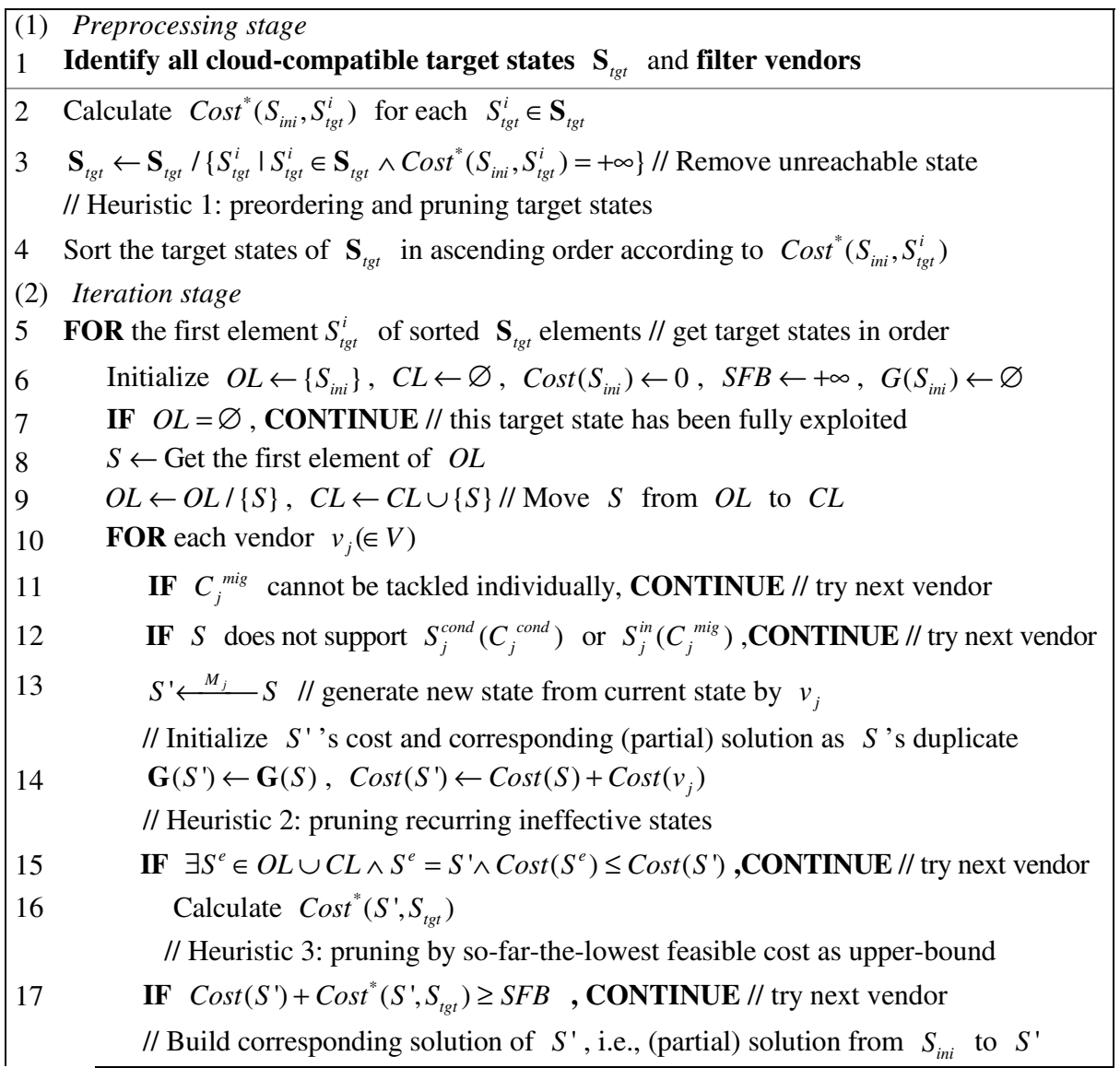




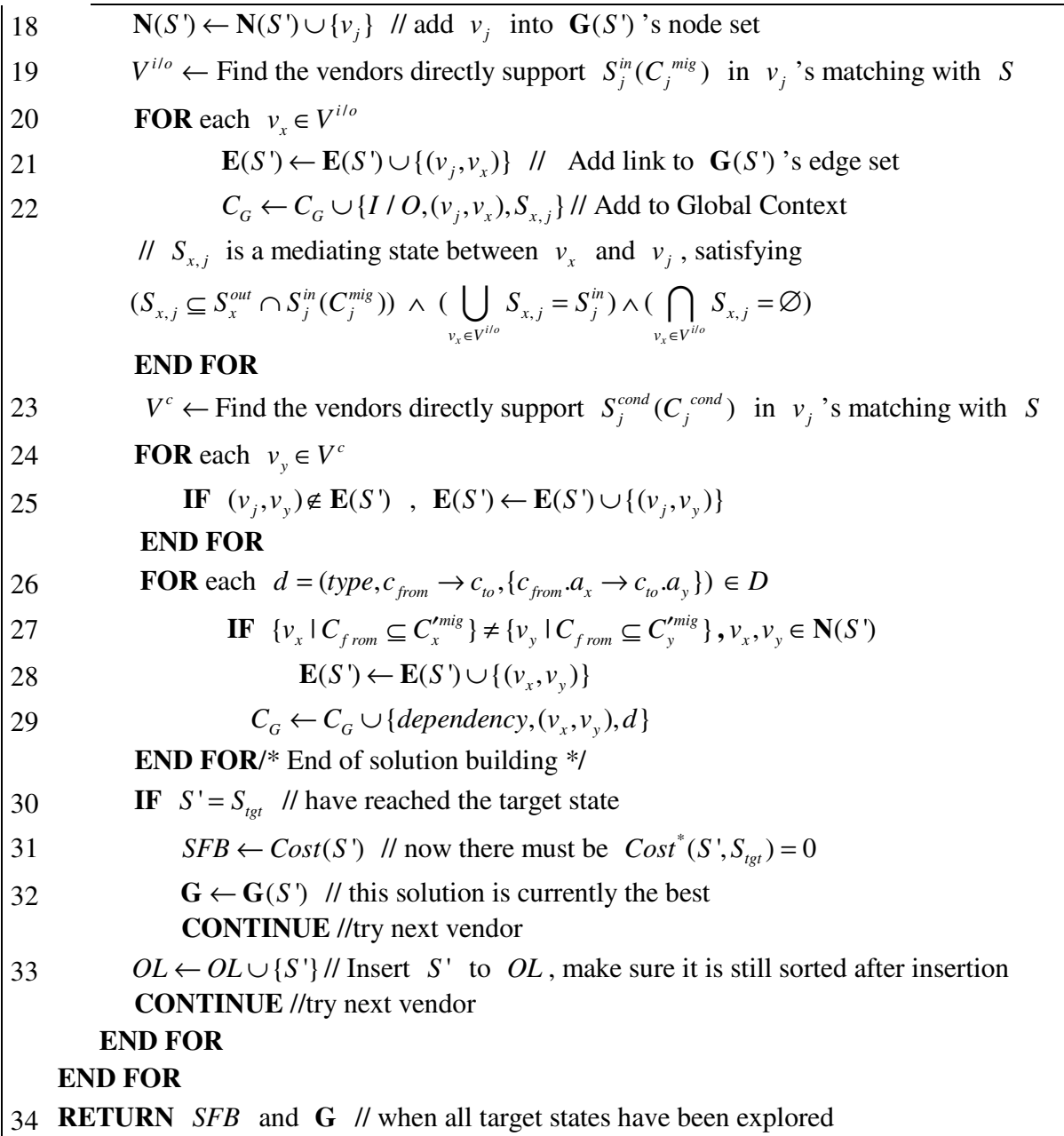

\section{Implementation and Evaluation}

In this section, we validated our approach via synthetic trace driven simulations. The settings of key parameters are shown in Table 1. Based on the parameter settings, we randomly generated a cloud profile from which two target states are identified. We evaluated the performance of the algorithm in term of computation time consumed to obtain the optimal solution under different conditions.

Table 1. Parameter settings for performance evaluation

\begin{tabular}{|c|c|c|}
\hline Type & Parameter & (range of) value \\
\hline variable & \# of heterogeneous servers per application $(\mathrm{NoHS})$ & $\mathbf{1} 16$ \\
\hline variable & \# of components per server $(\mathrm{NoC})$ & $\mathbf{6} 14$ \\
\hline variable & \# of vendors per component type $(\mathrm{NoV})$ & $\mathbf{5 , 1 0 , 1 5}$ \\
\hline
\end{tabular}


Fig.1(a) shows the computation time of the algorithm with respect to NoHS and $N o V$, under $N o C=10$. As can be seen, by adopting multiple effective pruning strategies, the computation time of the algorithm is controlled within a reasonable scale. Although the increasing speed of computation time tends to increase as scale of the synthetic trace grows, the algorithm is proved to be able to handle scenarios with a sufficiently large problem scale efficiently, i.e., $15 *(1+12+12 * 10)=1995$ vendors when $N o V=15$, which is far more than existing vendors in realistic market. Under this large scale scenario, our algorithm managed to find the optimal composition solution within 25 minutes. This performance is apparently affordable in practical uses, given that a real migration planning could usually take several hours or even days.

Fig.1(b) shows the computation time with respect to $\mathrm{NoC}$ and $\mathrm{NoV}$, where $\mathrm{NoHS}$ is set to 10 . The results show that the computation time with respect to $\mathrm{NoC}$ increases much slower than that w.r.t $\mathrm{NoHS}$. Within certain scope, the curves are nearly linear, e.g., when $\mathrm{NoC}$ varying between 8 and 12 with $\mathrm{NoV}=15$, and $\mathrm{NoC}$ varying between 10 and 14 with $N o V=10$. These results reveal the fact that $N o H S$ is inherently more influential to the performance of the algorithm, since each new heterogeneous server is likely to bring much more new types of intra-server components, which further increases the complexity of the problem.

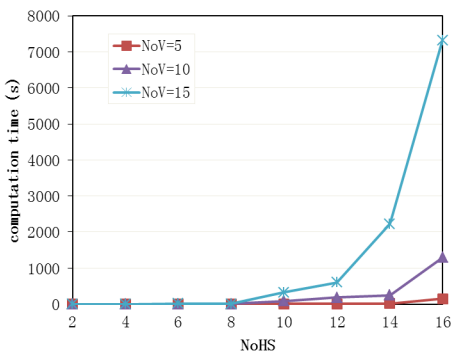

(a) computation time w.r.t. $\mathrm{NoHS}$ and $\mathrm{NoV}$

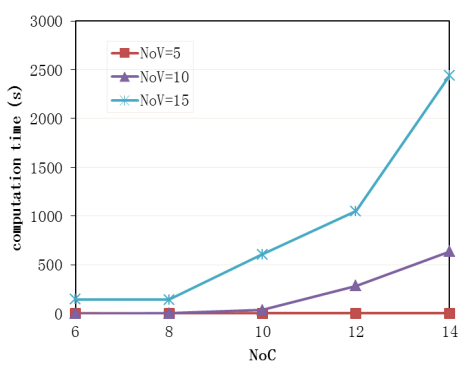

(b) computation time w.r.t. $\mathrm{NoC}$ and $\mathrm{NoV}$

Fig. 1. Algorithm performance w.r.t. $\mathrm{NoHS}, \mathrm{NoC}$ and $\mathrm{NoV}$

Based on the above performance analysis with the simulated representative synthetic data, we can conclude that the optimization model and algorithm are both effective and efficient to be applied in the real business application migration cases.

\section{Related Work}

Migrating application to cloud has been widely studied from different perspectives, which include frameworks and tools for migration execution [9], toolkits and models to support decision making of migration feasibility [10], frameworks for target cloud selection [11], techniques to discover application configuration for migration [12], and etc. Specifically, in [9] the authors introduced the Darwin framework which integrates set of tools that can enable smooth workload migration to cloud or noncloud environment. In [10], the authors proposed Cloud Transformation Advisor which recommends an optimal application transformation solution based on transforming patterns definition. However, all of these works only focused on 
particular application types, software stacks or target clouds and are hardly applied in migrating applications with complicated topologies and software stacks individually.

Though service composition has been widely studied in services computing, being orthogonal with these existing works, in this paper, we apply service composition to solve the realistic and urgent challenges in cloud migration. To our best knowledge, this is the first work proposing to solve the cloud migration problem by consolidating different vendor services.

\section{Conclusion and Future Work}

In this paper, we presented a novel service composition based approach for business application migration to cloud to accomplish the complicated migration tasks at lower cost, which consolidates diverse capabilities offered by different migration vendors. By capturing and formalizing the key concepts involved in cloud migration, we formally modeled the problem and provided an efficient algorithm to solve its optimal problem. Through synthetic trace driven simulations, the effectiveness and efficiency of the modeling and algorithm have been validated. Besides the cost issue during consolidating vendor services, we plan to conduct more factors such as network performance and user preference in the future.

\section{References}

[1] Ahorlu, M.: European Cloud Professional Services, Cloud Management Services, and Hosted Private Cloud 2012-2016 Forecast. In: IDC Market Analysis (November 2012)

[2] Racemi, http: //www.racemi.com/

[3] Cohesive Flexible Technologies, http: / / www. cohesiveft.com/

[4] AppZero, http: / /www . appzero.com/

[5] CliQr Technologies, http: / / www.cliqr.com/

[6] Ravello Systems, http: / / www . ravellosystems.com/

[7] Frey, S., Hasselbring, W.: Model-Based Migration of Legacy Software Systems to Scalable and Resource-Efficient Cloud-Based Applications: The CloudMIG Approach. In: Intl Conf. on Cloud Computing, GRIDs, and Virtualization (November 2010)

[8] IBM SmartCloud Enterprise, http: / / www-935.ibm.com/services/us/en/cloud-enterprise/

[9] Ward, C., et al.: Workload Migration into Clouds - Challenges, Experiences, Opportunities. In: Proc. of Intl. Conf. on Cloud Computing, pp. 164-171 (June 2010)

[10] Chee, Y., Zhou, N., Meng, F.J., Bagheri, S., Zhong, P.: A Pattern-Based Approach to Cloud Transformation. In: Proc. of Intl. Conf. on Cloud Computing, pp. 388-395 (2011)

[11] Khajeh-Hosseini, A., Sommerville, I., Bogaerts, J., Teregowda, P.: Decision Support Tools for Cloud Migration in the Enterprise. In: Intl Conf. on Cloud Computing, pp. 541-548 (June 2011)

[12] Menzel, M., Ranjan, R.: CloudGenius: Decision Support for Web Server Cloud Migration. In: Intl World Wide Web Conference, pp. 979-988 (April 2012) 